\title{
JVL im Wandel - innovatives Format in neuer Gestalt
}

\author{
Nico Ruf · Saskia Dombrowski
}

(C) Bundesamt für Verbraucherschutz und Lebensmittelsicherheit (BVL) 2012

Das Journal für Verbraucherschutz und Lebensmittelsicherheit (JVL) geht mit neuen Ideen in den aktuellen Band. In diesem Jahr erscheint die Zeitschrift bereits im siebten Jahrgang und damit es nicht eintönig oder gar verflixt wird, haben wir frischen Wind in die Heftgestaltung gebracht. Selbstverständlich finden relevante Inhalte aus dem gesamten Kaleidoskop des Verbraucherschutzes - vom Acker bis zum Teller weiterhin ihren Platz im JVL. Die Aufteilung der Beiträge in Leitthema und generelle Aspekte entfällt jedoch - zugunsten einer Zuordnung der Artikel nach der Art der dargestellten Inhalte.

Das JVL war von der ersten Ausgabe an ein innovatives Format, welches begutachtete Artikel sowie Mitteilungen aus dem behördlichen Kontext zu den aktuellen Themen des Verbraucherschutzes in sich vereint. Aus dieser Kombination entstehen die Besonderheit des JVL und sein hoher Wert für die behördenassoziierte Kommunikation. Dass dieses Format erfolgreich funktioniert, zeigen die weiter steigenden Zahlen an Einreichungen und Lesern sowie seine Listung bei Thomson ISI (Institute for Scientific Information) seit 2009 mit einem Impact Factor. Diese besondere Charakteristik des JVL wird auch in der Zweisprachigkeit der Artikel, die teilweise in deutscher und englischer Sprache verfasst sind, deutlich. Die Tendenz für Einreichungen

N. Ruf · S. Dombrowski ( $\square)$

Bundesamt für Verbraucherschutz und

Lebensmittelsicherheit, Mauerstr. 39-42, 10117 Berlin,

Germany

e-mail: jvl@bvl.bund.de in englischer Sprache und ausländischer Autoren nimmt aktuell stark zu.

Der Anspruch des JVL, den Anforderungen seriöser Wissenschaftskommunikation sowie behördlicher Kommunikation gerecht zu werden, besteht auch im neuen Jahrgang selbstverständlich fort. Es gilt mehr denn je, den Themen des Risikomanagement und des Verbraucherschutzes rund um den Zuständigkeitsbereich des Bundesamtes für Verbraucherschutz und Lebensmittelsicherheit (BVL) ein lebendiges Forum und den Lesern wissenschaftlich hochwertige Qualität in Form begutachteter Artikel zu bieten.

Mit der in der vorliegenden Ausgabe beginnenden, etwas gewandelten Gestalt entfällt die Zuordnung eines großen Teils der Artikel als genereller Aspekt. Die Artikel werden vielmehr den Standards wissenschaftlicher Zeitschriften und damit den Gewohnheiten der Leser entsprechend nun in RESEARCH ARTICLE (Originalartikel), REVIEW ARTICLE (Übersichtsartikel), SHORT COMMUNICATIONS (Kurzbeiträge) und METHODS (Methodenartikel) gruppiert.

So bezieht sich das Titelbild der vorliegenden Ausgabe auf einen Übersichtsartikel von Bavorová und Hirschauer, welcher verschiedene Systeme der amtlichen Lebensmittelüberwachung und die Nachvollziehbarkeit ihrer Ergebnisse sowie die sich ergebenden Konsequenzen für die kontrollierten Betriebe und den Verbraucher in Dänemark und Deutschland vergleicht. Gegenstand ist unter anderem das im Frühjahr 2009 vom Berliner Bezirk Pankow eingeführte Smiley-System zur Veröffentlichung amtlicher Kontrollergebnisse der Lebensmittelbetriebe auf der Basis des Verbraucherinformationsgesetzes. Der Pankow-Smiley hat sowohl in Berlin als auch bundesweit für großes Interesse gesorgt und wird 
neben dem seit Juli 2011 eingeführten einheitlichen System in Berlin (http://www.berlin.de/sicher-essen) in Erweiterung dessen fortgeführt.

Im zweiten Übersichtsartikel dieser Ausgabe stellen die Autoren Kühn und Lehrach vom Berliner Max-Planck-Institut für Molekulargenetik einen integrierten Ansatz von moderner Genom-Sequenzierung und komplexen bioinformatischen Methoden für eine individualisierte Krebsdiagnostik und -therapie vor.

Die weiteren Artikel in dieser Ausgabe unter der Rubrik RESEARCH ARTICLE bilden sehr eindrucksvoll das umfangreiche Portfolio des JVL ab: Ruoff et al. präsentieren eine Dioxin-Analyse verschiedener Milchprodukte aus Deutschland. Halle et al. untersuchten den Einfluss von mit konjugierten Linolsäuren angereicherte Futtermittel auf das Wachstum von Geflügel und die Fettzusammensetzung im Geflügelfleisch. Näumann et al. berichten in ihrer Studie über die Bienenkrankheit „Amerikanische Faulbrut" und über Rückstände des verursachenden Erregers sowie verschiedener Arzneimittel in importierten Honig. Strauß et al. untersuchen die Keimfähigkeit von Samen verschiedener Nutzpflanzen nach unterschiedlichen Verweilzeiten und Umgebungsbedingungen in Biogasanlagen. Die Reihe wird ergänzt durch den Artikel von Finke et al. aus dem Bereich Epigenetik der Pflanzen, die mit Hilfe von kleinen, nicht-kodierenden RNS-Molekülen bestimmte Abschnitte der pflanzlichen DNS durch De-novo-Methylierung inaktivieren.

Der erste Methodenartikel im JVL von Gürtler et al. beschreibt eine neue molekulargenetische
Nachweismethode für Kefeng 6, eine in der EU nicht zugelassene gentechnisch veränderte Reissorte.

Neben der Veröffentlichung von Forschungsergebnissen aus dem Zuständigkeitsbereich des BVL sind die amtlichen Mitteilungen ein fester inhaltlicher Bestandteil des JVL. Im neuen Konzept bestehen diese als Teil der ANNOUNCEMENTS and REPORTS weiter. Zahlreiche Mitteilungen aus dem relevanten Zeitraum (4. Quartal 2011) und den Bereichen Lebensmittel, Tabak und Tierarzneimittel sowie die im Rahmen der 98. Sitzung des Arbeitskreises Lebensmittelchemischer Sachverständiger der Länder und des Bundesamtes für Verbraucherschutz und Lebensmittelsicherheit (ALS) beschlossenen fachlichen Stellungnahmen finden sich in dieser Ausgabe. Darüber hinaus stellt die Bund/Länder-AG Gentechnik (LAG) zwei verabschiedete Methoden - zum einen zur molekularbiologischen Identifizierung von Pilzen mittels ITS-PCR und nachfolgender Sequenzierung und zum anderen zur Überprüfung der Spezies und Reinheit von Zelllinien mittels Multiplex-PCR - vor.

Mit der Rubrik EVENTS and NEWS rundet das JVL sein Angebot ab. An dieser Stelle finden sich sporadisch Besprechungen relevanter Buchtitel sowie Veranstaltungshinweise.

Um die Zeitschrift entlang aktueller Themenschwerpunkte redaktionell zu gestalten und Highlights $\mathrm{zu}$ setzen wird die Redaktion des JVL weiterhin Autoren einladen, Artikel zu spezifischen Themen einzureichen. Und: Last but not least wünschen wir unseren Lesern gewohntes Lesevergnügen in neuer Sortierung. 